\title{
A Novel Scoring System for Diagnosing Acute Mesenteric Ischemia in the Emergency Ward: Methodological Issues: Reply
}

\author{
Zhen Wang ${ }^{1}$
}

Published online: 20 October 2017

(C) Société Internationale de Chirurgie 2017

Dear Editors,

Thanks for your e-mail. I have read the letter from Safiri and Ayubi [1]. The following is my reply.

Firstly, I am very appreciated for Saeid's and Erfan's rigorously scientific attitude and attention to our study. And thanks very much for their suggestions. I have considered their questions very carefully. Indeed, we did not consider all the problems on statistics in our manuscript when drafting it.

1. "Bootstrapping method": we were not familiar with this statistical method before. Indeed, this is the limitation of our study in dealing with the data.

2. "In the standard model construction strategy, the multicollinearity between the candidate variables must be checked in the first step; then, variables with $P$ value $<0.2$ are imported into the multivariable model". This was what we did when dealing with our data. Maybe we did not elucidate these detailed enough in the part of statistical analysis, but according to Table 2, we did deal with our data as Saeid's and Erfan's suggestion.
3. "Sparse data bias" According to Saeid's and Erfan's suggestion, we read the study by Greenland et al. (Greenland S, Mansournia MA, Altman DG: Sparse data bias: a problem hiding in plain sight. bmj 2016, 352:i1981.) and examined our data carefully. According to "preliminary checks for sparse data bias" mentioned in the study, we do not think there is sparse data bias in our study.

Thanks very much! If any problem exists, please let me know.

Best wishes,

Zhen Wang

\section{References}

1. Safiri S, Ayubi E (2017) A novel scoring system for diagnosing acute mesenteric ischemia in the emergency ward: methodological issues. World J Surg. doi:10.1007/s00268-017-4216-Z
Zhen Wang

wangzhensurgeon@163.com

1 Department of Gastrointestinal Surgery, The First Affiliated Hospital of Guangxi Medical University, Nanning, China 